\title{
MicroRNA expression profiling during the suckling-to-weaning transition in pigs
}

\author{
Hyun Jun Jang ${ }^{1}$ and Sang In Lee ${ }^{2 *}$ \\ ${ }^{1}$ Center for Industrialization of Agricultural and Livestock Microorganisms, Jeongeup 56212, Korea \\ ${ }^{2}$ Department of Animal Biotechnology, Kyungpook National University, Sangju 37224, Korea
}

Received: Jan 11, 2021

Revised: Apr 21, 2021

Accepted: May 1, 2021

*Corresponding author

Sang In Lee

Department of Animal Biotechnology,

Kyungpook National University, Sangju

37224, Korea.

Tel: +82-54-530-1943

E-mail: silee78@knu.ac.kr

Copyright $\odot 2021$ Korean Society of Animal Sciences and Technology.

This is an Open Access article distributed under the terms of the

Creative Commons Attribution

Non-Commercial License (http://

creativecommons.org/licenses/by-

nc/4.0/) which permits unrestricted

non-commercial use, distribution, and

reproduction in any medium, provided

the original work is properly cited.

ORCID

Hyun Jun Jang

https://orcid.org/0000-0003-2906-7543

Sang In Lee

https://orcid.org/0000-0002-0019-1834

Competing interests

No potential conflict of interest relevant

to this article was reported.

Funding sources

This research was supported by

Kyungpook National University

Research Fund, 2019.

Acknowledgements

Not applicable.

Availability of data and material Upon reasonable request, the datasets of this study can be available from the corresponding author.

\section{Abstract}

Weaning induces physiological changes in intestinal development that affect pigs' growth performance and susceptibility to disease. As a posttranscriptional regulator, microRNAs (miRNAs) regulate cellular homeostasis during intestinal development. We performed small RNA expression profiling in the small intestine of piglets before weaning (BW), 1 week after weaning $(1 \mathrm{~W})$, and 2 weeks after weaning $(2 \mathrm{~W})$ to identify weaning-associated differentially expressed miRNAs. We identified 38 differentially expressed miRNAs with varying expression levels among $\mathrm{BW}, 1 \mathrm{~W}$, and $2 \mathrm{~W}$. Then, we classified expression patterns of the identified miRNAs into four types. ssc-miR-196a and ssc-miR-451 represent pattern 1, which had an increased expression at $1 \mathrm{~W}$ and a decreased expression at 2W. ssc-miR-499-5p represents pattern 2, which had an increased expression at $1 \mathrm{~W}$ and a stable expression at $2 \mathrm{~W}$. ssc-miR7135-3p and ssc-miR-144 represent pattern 3, which had a stable expression at $1 \mathrm{~W}$ and a decreased expression at 2W. Eleven miRNAs (ssc-miR-542-3p, ssc-miR-214, ssc-miR-758, ssc-miR-4331, ssc-miR-105-1, ssc-miR-1285, ssc-miR-10a-5p, ssc-miR-4332, ssc-miR-503, ssc-miR-6782-3p, and ssc-miR-424-5p) represent pattern 4, which had a decreased expression at $1 \mathrm{~W}$ and a stable expression at $2 \mathrm{~W}$. Moreover, we identified 133 candidate targets for miR-196a using a target prediction database. Gene ontology and Kyoto Encyclopedia of Genes and Genomes (KEGG) pathway analyses revealed that the target genes were associated with 19 biological processes, 4 cellular components, 8 molecular functions, and 7 KEGG pathways, including anterior/posterior pattern specification as well as the cancer, PI3K-Akt, MAPK, GnRH, and neurotrophin signaling pathways. These findings suggest that miRNAs regulate the development of the small intestine during the weaning process in piglets by anterior/posterior pattern specification as well as the cancer, PI3K-Akt, MAPK, GnRH, and neurotrophin signaling pathways.

Keywords: miRNA, Small RNA sequencing, Weaning pigs

\section{INTRODUCTION}

The suckling-to-weaning transition is an extremely stressful event in mammals, including pigs. During this transitional period, a pig is subjected to a number of environmental changes, including removal from the sow and littermates, the fighting and establishment of social hierarchy, transportation stress, 
Authors' contributions Conceptualization: Lee SI.

Data curation: Jang HJ, Lee SI.

Formal analysis: Jang HJ, Lee SI.

Methodology: Jang HJ, Lee SI.

Software: Jang HJ, Lee SI.

Validation: Jang HJ, Lee SI.

Writing - original draft: Jang HJ, Lee SI.

Ethics approval and consent to participate The animal care and experimental protocols of the present study were approved by the Animal Care, and Use Committee of Dankook University (IAUCU protocol No. DKU-19-024) and all methods were performed in accordance with the relevant guidelines and regulations. abrupt transitions in diet, and a change of temperature and water [1]. These environmental stresses during weaning lead to immune dysfunctions in pigs, causing reduced feed intake and growth as well as decreased health [1,2]. Moreover, reduced feed intake and growth as well as decreased health during the suckling-to-weaning transition are influenced by acute physiological changes, including the secretion of digestive enzymes and structural disruption in the gastrointestinal tract [3]. Thus, it is important to address the dysfunction of the gastrointestinal tract in pigs as quickly as possible to maximize productivities, including growth.

The small intestine has three major functions: 1) the absorption of nutrients, including amino acids, fatty acids, and monosaccharides, from digested proteins, lipids, and carbohydrates [4];2) intestinal barrier function to prevent the passage of harmful bacteria and toxins [5]; and 3) the maintenance of lumen viscosity via the secretion or absorption of water and electrolytes [6]. Thus, intestinal dysfunction directly induces growth retardation and increased susceptibility to infections. Among the causes of intestinal dysfunction, the suckling-to-weaning transition directly affects the structural disruption of the intestine, inducing diarrhea [7]. Thus, it is essential to maintain proper intestinal function for growth and health during the suckling-to-weaning transition of pigs.

MicroRNAs (miRNAs) are small noncoding RNAs with a length of 18-26 nucleotides; they regulate gene expression by binding to the 3'untranslated region (3'UTR) of target mRNAs [8]. It is well-accepted that miRNAs play critical roles in nearly all biological processes, including cellular proliferation, differentiation, apoptosis, disease, and development [9]. Moreover, studies have reported that miRNAs regulate cellular homeostasis, including proliferation, differentiation, and apoptosis, during intestinal development [10,11].

Thus, to identify miRNAs that play critical roles on intestinal dysfunction during the sucklingto-weaning transition in pigs, we evaluated miRNA expression profiles during the suckling-toweaning transition in pigs. In addition, we identified candidate targets for differentially expressed miRNAs and analyzed gene ontology (GO) and Kyoto Encyclopedia of Genes and Genomes (KEGG) pathways for the functional categorization of target genes.

\section{MATERIAL AND METHODS}

\section{Animals and sample preparation}

Crossbred ([Yorkshire $\times$ Landrace] $\times$ Duroc) pigs were housed in an environmentally controlled room with a slatted plastic floor. Nine piglets $(\mathrm{n}=3$, each group) were sedated using xylazine and ketamine and then euthanized with an overdose of pentobarbital administered via an ear vein before weaning $(\mathrm{BW}), 1$ week after weaning $(1 \mathrm{~W})$, and 2 weeks after weaning $(2 \mathrm{~W})$. The abdominal cavity of each piglet was opened, and small intestine samples were collected. All intestine samples were frozen in liquid nitrogen and stored at $-80^{\circ} \mathrm{C}$. Total RNA was isolated using TRIzol reagent (Invitrogen, Carlsbad, CA, USA). RNA quality was assessed using Agilent 2100 Bioanalyzer with RNA 6000 Pico chip (Agilent Technologies, Santa Clara, CA, USA). RNA was quantified using NanoDrop 2000 spectrophotometer system (Thermo Fisher Scientific, Waltham, MA, USA).

\section{Library preparation and sequencing}

Library was constructed using NEBNext Multiplex Small RNA Library Prep kit (New England BioLabs, Ipswich, MA, USA) as per the manufacturer's instructions for the control and test RNAs. In brief, for library construction, $1 \mu \mathrm{g}$ of total RNA from each sample was used to ligate the adaptors, and cDNA was then synthesized using reverse transcriptase with adaptor-specific primers. Polymerase chain reaction (PCR) was performed for library amplification, and a clean-up of the libraries was performed using QIAquick PCR Purification kit (Qiagen, Hilden, German) 
and AMPure XP beads (Beckmancoulter, Brea, CA, USA). The yield and size distribution of the small RNA libraries were assessed using Agilent 2100 Bioanalyzer instrument for a high-sensitivity DNA assay (Agilent Technologies). High-throughput sequences were produced using NextSeq500 system by way of single-end 75 bp sequencing (Illumina, San Diego, CA, USA).

\section{Data analysis}

Sequence reads were mapped using bowtie2 software tool to obtain .bam files (an alignment file format). A mature miRNA sequence was used as a reference for mapping. Read counts mapped on the mature miRNA sequence were extracted from the alignment file using bedtools (v2.25.0) and Bioconductor with $\mathrm{R}$ statistical programming language (version 3.2.2). The read counts were then used to determine the expression level of miRNAs. The quantile normalization method was utilized for comparison between samples.

\section{Target prediction and target gene annotation}

The target genes of the selected miRNAs were predicted using miRDB database (http://www. mirdb.org/). The functional annotation tool DAVID (http://david.ncifcrf.gov/) was used for GO and KEGG pathway analysis of the predicted target genes. The data was retrieved, and a $p$-value of $<0.05$ was considered a statistically significant difference.

\section{RESULTS}

\section{Identification of differentially expressed miRNAs}

We performed a high-throughput sequencing of small RNA isolated from the small intestine tissue of piglets at BW, $1 \mathrm{~W}$, and $2 \mathrm{~W}$ (Fig. 1) to identify differentially expressed miRNAs at different time-points. We found 19 differentially expressed miRNAs with varied expression levels between $\mathrm{BW}$ and $1 \mathrm{~W}$, of which 6 were upregulated and 13 were downregulated at $1 \mathrm{~W}$ (Table 1). Likewise, 28 differentially expressed miRNAs were identified between $2 \mathrm{~W}$ and BW, of which 9 were upregulated and 19 were downregulated (Table 2). Moreover, there were seven differentially expressed miRNAs between $2 \mathrm{~W}$ and $1 \mathrm{~W}$, all of which were downregulated (Table 3). Finally, a total of 38 differentially expressed miRNAs with different expression levels were found among BW, $1 \mathrm{~W}$, and $2 \mathrm{~W}$; of these, 12 were upregulated and 26 were downregulated across the 3 time-points

Threshold line: 2 fold
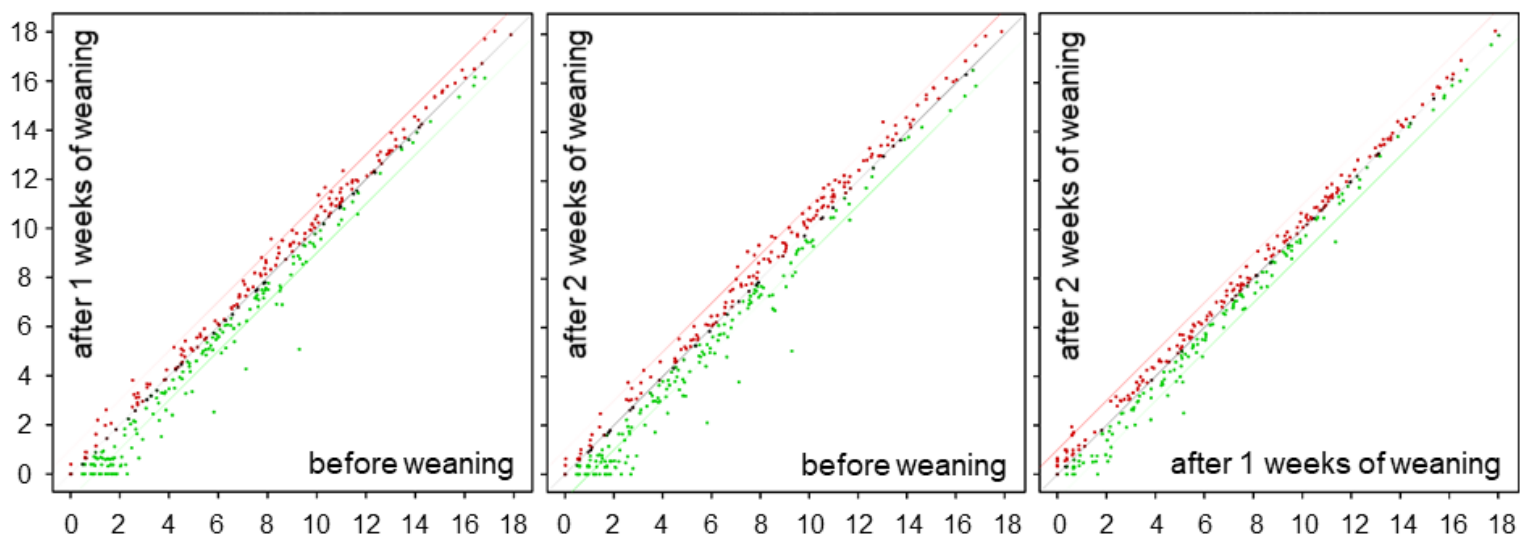

Fig. 1. Scatter plot of small RNA sequencing. The scatter plot is useful for assessing variation among samples. The axes of the scatter plot are the samples' fold-change (the ratio scale). 
Table 1. Differentially expressed miRNAs in small intestine at before weaning and after 1 week of weaning

\begin{tabular}{|c|c|c|c|c|c|}
\hline \multirow{2}{*}{ No. } & \multirow{2}{*}{ Gene symbol } & \multirow{2}{*}{ Fold change } & \multirow{2}{*}{$p$-value } & \multicolumn{2}{|c|}{ Average of normalized read count (log2) } \\
\hline & & & & Before weaning & After $1 \mathrm{~W}$ \\
\hline 1 & ssc-miR-96-5p & 2.67 & 0.04 & 8.15 & 9.57 \\
\hline 2 & ssc-miR-183 & 2.52 & 0.01 & 10.33 & 11.67 \\
\hline 3 & ssc-miR-196a & 2.45 & 0.02 & 2.52 & 3.82 \\
\hline 4 & ssc-miR-451 & 2.44 & 0.01 & 10.07 & 11.36 \\
\hline 5 & ssc-miR-182 & 2.43 & 0.04 & 11.06 & 12.34 \\
\hline 6 & ssc-miR-499-5p & 2.07 & 0.02 & 7.76 & 8.81 \\
\hline 7 & ssc-miR-542-3p & 0.48 & 0.03 & 8.51 & 7.45 \\
\hline 8 & ssc-miR-10a-5p & 0.47 & 0.02 & 11.66 & 10.58 \\
\hline 9 & ssc-miR-503 & 0.43 & 0.04 & 6.13 & 4.92 \\
\hline 10 & ssc-miR-424-5p & 0.41 & 0.00 & 6.66 & 5.38 \\
\hline 11 & ssc-miR-758 & 0.38 & 0.01 & 4.68 & 3.28 \\
\hline 12 & ssc-miR-493-3p & 0.37 & 0.01 & 3.61 & 2.18 \\
\hline 13 & ssc-miR-214 & 0.36 & 0.00 & 8.37 & 6.91 \\
\hline 14 & ssc-miR-105-1 & 0.33 & 0.03 & 4.94 & 3.35 \\
\hline 15 & ssc-miR-1285 & 0.31 & 0.02 & 8.60 & 6.89 \\
\hline 16 & ssc-miR-184 & 0.30 & 0.03 & 4.14 & 2.40 \\
\hline 17 & ssc-miR-4332 & 0.14 & 0.01 & 7.13 & 4.28 \\
\hline 18 & ssc-miR-6782-3p & 0.10 & 0.01 & 5.83 & 2.52 \\
\hline 19 & ssc-miR-4331 & 0.05 & 0.01 & 9.29 & 5.08 \\
\hline
\end{tabular}

1W, 1 week after weaning

(Fig. 2A). Among the 12 upregulated miRNAs, 3 miRNAs (ssc-miR-96-5p, ssc-miR-183, and sscmiR-182) were upregulated at $1 \mathrm{~W}$ compared with BW (Fig. 2B) and 8 miRNAs (ssc-miR-29b, ssc-miR-34c, ssc-miR-29c, ssc-miR-29a, ssc-miR-122, ssc-miR-22-3p, ssc-miR-221-5p, and sscmiR-423-5p) were upregulated at $2 \mathrm{~W}$ compared with BW. No changed miRNAs were identified at $2 \mathrm{~W}$ compared with $1 \mathrm{~W}$. In addition, among the 26 downregulated miRNAs, 2 miRNAs (sscmiR-493-3p and ssc-miR-184) were downregulated at $1 \mathrm{~W}$ compared with BW (Fig. 2B), 6 miRNAs (ssc-miR-450b-5p, ssc-miR-455-5p, ssc-miR-10a-3p, ssc-miR-450c-5p, ssc-miR-450a, and ssc-miR-135) were downregulated at $2 \mathrm{~W}$ compared with BW, and 3 miRNAs (ssc-miR-1277, ssc-miR-212, and ssc-miR-224) were downregulated at $2 \mathrm{~W}$ compared with $1 \mathrm{~W}$. From these results, it is clear that the suckling-to-weaning transition significantly affects miRNA expression levels in the small intestine.

\section{Expression patterns of differentially expressed miRNAs}

We identified 4 types of expression patterns from the 16 differentially expressed miRNAs that were commonly expressed during the weaning process (Fig. 3). Pattern 1 is represented by sscmiR-196a and ssc-miR-451, which showed a pattern of increased expression at $1 \mathrm{~W}$ and decreased expression at $2 \mathrm{~W}$. Pattern 2 is represented by ssc-miR-499-5p, with an increased expression at $1 \mathrm{~W}$ and a stable expression at $2 \mathrm{~W}$. Pattern 3 is represented by ssc-miR-7135-3p and ssc-miR-144, with a stable expression at $1 \mathrm{~W}$ and a decreased expression at $2 \mathrm{~W}$. Eleven miRNAs (ssc-miR-542-3p, ssc-miR-214, ssc-miR-758, ssc-miR-4331, ssc-miR-105-1, ssc-miR-1285, ssc-miR-10a-5p, sscmiR-4332, ssc-miR-503, ssc-miR-6782-3p, and ssc-miR-424-5p) represent pattern 4, showing a decreased expression at $1 \mathrm{~W}$ and a stable expression at 2W. We focused on ssc-miR-196a (pattern 1) for further analysis. 
Table 2. Differentially expressed miRNAs in small intestine at before weaning and after 2 week of weaning

\begin{tabular}{|c|c|c|c|c|c|}
\hline \multirow{2}{*}{ No. } & \multirow{2}{*}{ Gene symbol } & \multirow{2}{*}{ Fold change } & \multirow{2}{*}{$p$-value } & \multicolumn{2}{|c|}{ Average of normalized read count (log2 } \\
\hline & & & & Before weaning & After $\mathbf{2 W}$ \\
\hline 1 & ssc-miR-29b & 3.10 & 0.01 & 7.48 & 9.11 \\
\hline 2 & ssc-miR-34c & 2.69 & 0.01 & 7.94 & 9.37 \\
\hline 3 & ssc-miR-29c & 2.68 & 0.01 & 7.07 & 8.49 \\
\hline 4 & ssc-miR-29a & 2.62 & 0.01 & 13.02 & 14.41 \\
\hline 5 & ssc-miR-122 & 2.41 & 0.01 & 8.46 & 9.74 \\
\hline 6 & ssc-miR-499-5p & 2.31 & 0.01 & 7.76 & 8.97 \\
\hline 7 & ssc-miR-22-3p & 2.16 & 0.01 & 10.59 & 11.71 \\
\hline 8 & ssc-miR-221-5p & 2.10 & 0.01 & 8.61 & 9.68 \\
\hline 9 & ssc-miR-423-5p & 2.08 & 0.01 & 10.80 & 11.86 \\
\hline 10 & ssc-miR-424-5p & 0.49 & 0.01 & 6.66 & 5.62 \\
\hline 11 & ssc-miR-214 & 0.48 & 0.01 & 8.37 & 7.30 \\
\hline 12 & ssc-miR-450b-5p & 0.45 & 0.01 & 9.43 & 8.27 \\
\hline 13 & ssc-miR-542-3p & 0.44 & 0.02 & 8.51 & 7.33 \\
\hline 14 & ssc-miR-503 & 0.44 & 0.04 & 6.13 & 4.95 \\
\hline 15 & ssc-miR-455-5p & 0.44 & 0.01 & 9.32 & 8.12 \\
\hline 16 & ssc-miR-758 & 0.43 & 0.01 & 4.68 & 3.46 \\
\hline 17 & ssc-miR-10a-5p & 0.42 & 0.01 & 11.66 & 10.40 \\
\hline 18 & ssc-miR-10a-3p & 0.39 & 0.01 & 5.78 & 4.42 \\
\hline 19 & ssc-miR-7135-3p & 0.33 & 0.01 & 4.83 & 3.21 \\
\hline 20 & ssc-miR-144 & 0.31 & 0.01 & 4.20 & 2.50 \\
\hline 21 & ssc-miR-450c-5p & 0.30 & 0.01 & 8.51 & 6.78 \\
\hline 22 & ssc-miR-105-1 & 0.30 & 0.03 & 4.94 & 3.20 \\
\hline 23 & ssc-miR-450a & 0.29 & 0.01 & 8.54 & 6.74 \\
\hline 24 & ssc-miR-135 & 0.28 & 0.01 & 6.63 & 4.80 \\
\hline 25 & ssc-miR-1285 & 0.27 & 0.01 & 8.60 & 6.70 \\
\hline 26 & ssc-miR-4332 & 0.10 & 0.01 & 7.13 & 3.78 \\
\hline 27 & ssc-miR-6782-3p & 0.08 & 0.01 & 5.83 & 2.11 \\
\hline 28 & ssc-miR-4331 & 0.05 & 0.01 & 9.29 & 5.04 \\
\hline
\end{tabular}

2W, 2 weeks after weaning.

Table 3. Differentially expressed miRNAs in small intestine at after 1 week of weaning vs after 2 week of weaning

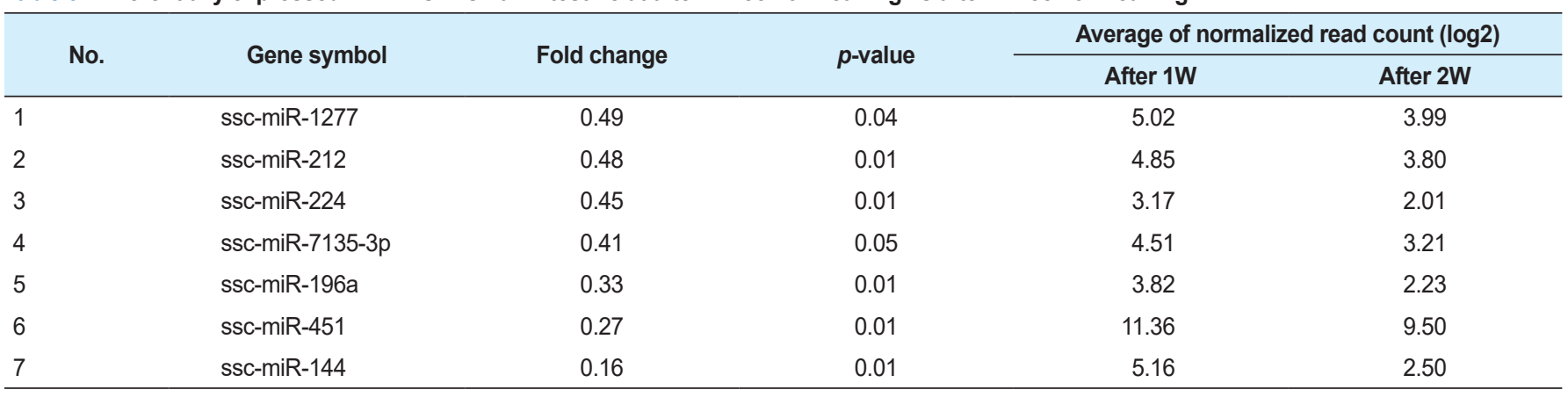

$1 \mathrm{~W}, 1$ week after weaning; $2 \mathrm{~W}, 2$ weeks after weaning. 
A

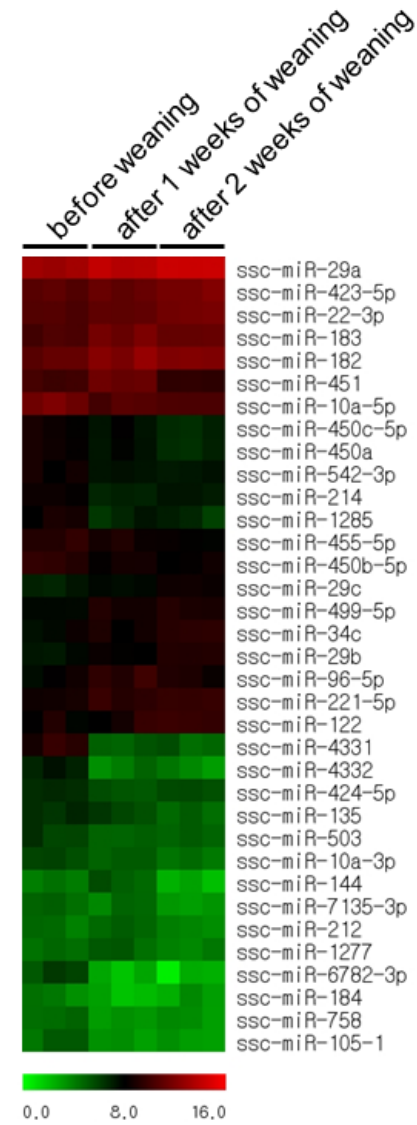

B

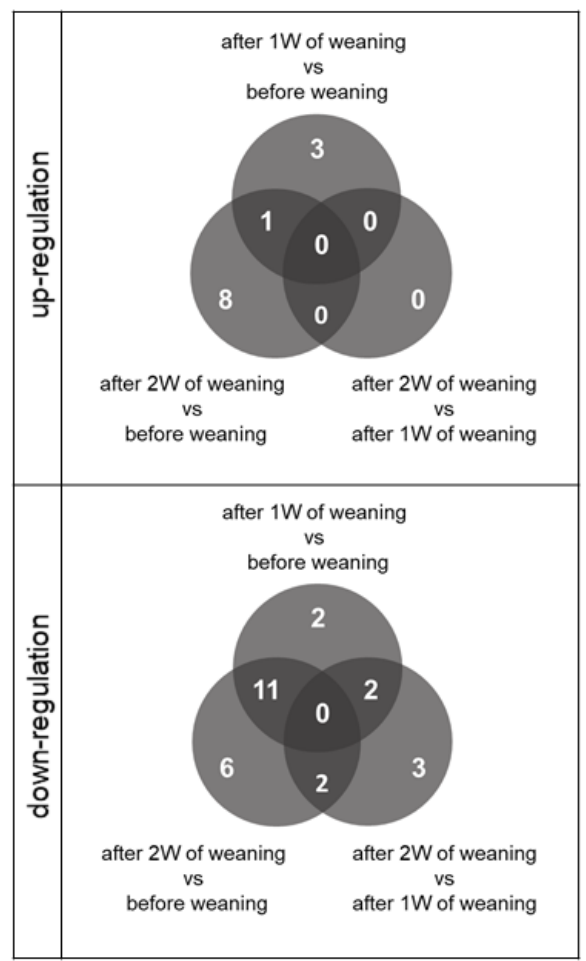

Fig. 2. Differentially expressed miRNAs among the three groups (before weaning, after 1 week of weaning, and after 2 weeks of weaning [ $n=3]$ ). (A) Hierarchical clustering of differentially expressed miRNAs. Red indicates high relative expression and green denotes low relative expression. miRNA with expression foldchange $>2.0$ was considered statistically significant. (B) Venn diagram of differentially expressed miRNAs that were up- or downregulated at least two-fold among the three samples.

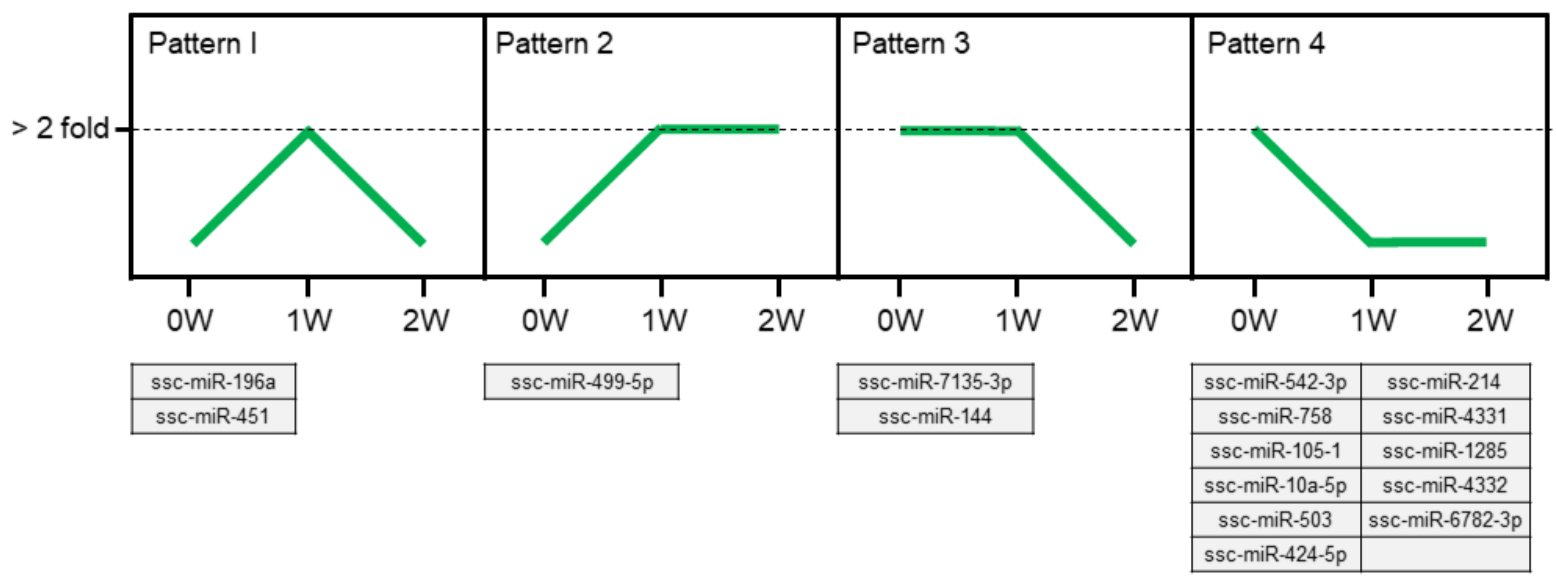

Fig. 3. Four unique expression patterns of differentially expressed miRNAs before weaning, after 1 week of weaning, and after 2 weeks of weaning. The displayed miRNA expression change is at least two-fold. 


\section{Candidate target prediction and target gene annotation}

The predicted target genes of miR-196a-5p were identified using the miRDB database. Because a target prediction database for pig is unavailable, we analyzed the prediction targets of human and mouse miR-196a-5p that represent the same miRNA sequence as that of pig miR-196a (Table 4). We found 370 and 296 candidate targets for hsa-miR-196a-5p and mmu-miR-196a-5p, respectively (Fig. 4A and Supplementary Tables S1 and S2). A total of 133 candidate genes were common between these candidate targets of hsa-miR-196a-5p and mmu-miR-196a-5p.

GO enrichment analysis using the DAVID database showed that the 133 common targets comprised 19 biological processes, 4 cellular components, and 8 molecular functions (Supplementary Table S3). The GO targets were associated with the negative regulation of transcription of the RNA polymerase II promoter, anterior/posterior pattern specification, the transforming growth factor beta receptor signaling pathway, zinc ion binding, and chromatin binding. KEGG pathway analysis revealed that the differentially expressed genes were significantly enriched in miRNAs for cancer pathway, the PI3K-Akt signaling pathway, the MAPK signaling pathway, choline metabolism in cancer, the GnRH signaling pathway, Epstein-Barr virus infection, and the neurotrophin signaling pathway (Fig. 4B).

\section{DISCUSSION}

Mammals, including pigs, experience the most physiologically, environmentally, and socially stressful changes during the weaning process, and these stresses can affect susceptibility to disease as well as productivity types, including growth rate, feed intake, and feed efficiency [1,12]. From a physiological viewpoint, the most extreme changes occur in the intestine [6]. The transition from liquid to solid feed leads to structural changes in the intestinal villi because of the lack of digestive enzyme secretion, excluding those for lactose, during the weaning process at 3 or 4 weeks after birth $[12,13]$. The dramatic change in intestinal villi structures can cause diarrhea during the weaning

Table 4. Sequence similarity of miR-196a between human, mouse and pig

\begin{tabular}{lll}
\hline \multicolumn{1}{c}{ Species } & \multicolumn{1}{c}{ miRNA name } & \multicolumn{1}{c}{ miRNA sequences } \\
\hline Pig & ssc-miR-196a & UAGGUAGUUUCAUGUUGUUGGG \\
Human & hsa-miR-196a-5p & UAGGUAGUUUCAUGUUGUUGGG \\
Mouse & mmu-miR-196a-5p & UAGGUAGUUUCAUGUUGUUGGG \\
\hline
\end{tabular}

A

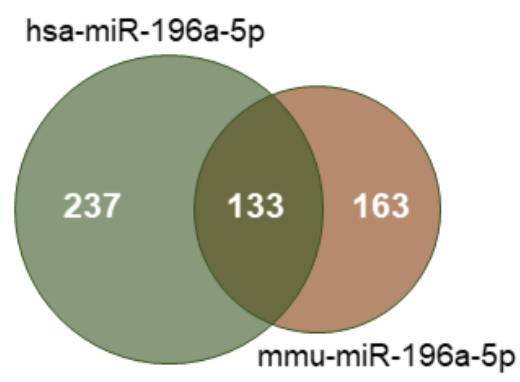

B

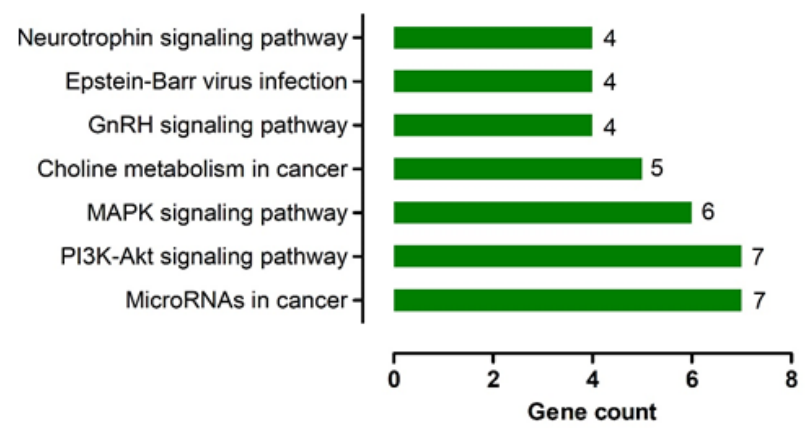

Fig. 4. Target gene prediction of miRNA196a. (A) Venn diagram of common target genes of hsa-miR-196A-5p and mmu-miR-196A-5p. (B) Kyoto Encyclopedia of Genes and Genomes pathway analysis of common target genes. 
process that directly affects intestinal barrier functionality, immunity, and wound healing processes $[4,14,15]$. Moreover, several studies have demonstrated that dramatic physiological changes induced by the weaning process affect gene expression profiles in intestinal tissues [16-19]. In the present study, we revealed that the weaning process affects miRNA expression profiles: 38 differentially expressed miRNAs had different expression levels across the 3 time-points studied (BW, 1W, and $2 \mathrm{~W}$ ). A previous report identified 136 differentially expressed miRNAs in weaning piglets at 1 , 4 , and 7 days after weaning from jejunal small RNA libraries [20], which is consistent with the findings of this study. Based on these findings, we suggest that the weaning process directly affects the expression profiles at the transcriptional and posttranscriptional levels.

The present study showed that intestinal development during the weaning process can affect miRNA expression profiles. miRNA, as a posttranscriptional regulator, modulates gene expression by binding to the $3^{\prime} \mathrm{UTR}$ of target mRNA in almost all cells, thereby affecting biological processes, development stages, and molecular functions. Moreover, studies have demonstrated that the development and biological functions of the intestine, including renewal, homeostasis, and inflammation, are regulated by miRNAs, as is the case with other tissues [21-24]. miR-34a and miR-320a strengthens intestinal barrier function by regulating tight-junction proteins in inflammatory bowel disease $[25,26]$. As was previously reported, miR-126 directly inhibits the expression of S1PR2, resulting in the impairment of the intestinal barrier function via the PI3KAKT signaling pathway [27]. The present study revealed that miR-196a expression was altered during the suckling-to-weaning transition and that miR-196a directly regulates target genes related to anterior/posterior pattern specification as well as the cancer, transforming growth factor beta receptor signaling, PI3K-Akt signaling, and MAPK signaling pathways in the development of small intestines during the weaning process. Based on these results, we suggest that miR-196a regulates the morphology and proliferative activity in the development of small intestines in piglets during the suckling-to-weaning transition. However, target validation assays for miR-196a are required for the accurate verification of targets and functions.

In the present study, we collected small intestine samples at different time-points $(\mathrm{BW}, 1 \mathrm{~W}$, and $2 \mathrm{~W}$ ) based on the functional changes of villous atrophy and crypt elongation after weaning [3]. Weaning induces the shortening of villi and increased crypt depth for approximately 1W [28,29]. Thus, the present study classified differentially expressed miRNAs at three time-points into four categories based on expression patterns. Of these four categories, we focused on pattern 1 as a critical time-point because of its increasing expression at $1 \mathrm{~W}$ and weakening expression at $2 \mathrm{~W}$. We showed that ssc-miR-196a and ssc-miR-451 represent pattern 1 in the present study among the 38 differentially expressed miRNAs. It is known that miR-451, which is a tumor marker for gastric and pancreatic cancer, promotes cell proliferation and metastasis [30,31]. Moreover, previous reports demonstrated that miR-196a promotes tumor growth and migration via signaling pathways, including the PTEN/Akt/FOXO1 pathway [32]. The present study revealed that miR196a regulates various signaling pathways, including those of PI3K-Akt, MAPK, and GnRH. Functional studies, including those on the knockdown or overexpression of miR-196a, are required in the future.

In conclusion, the present study identified 38 differentially expressed miRNAs using the highthroughput sequencing of small RNA isolated from the small intestine tissue of piglets during the weaning process. We showed that ssc-miR-196a and ssc-miR-451 show increasing expression at $1 \mathrm{~W}$ and weakening expression at $2 \mathrm{~W}$, which are critical time-points in piglet development. We expected that miR-196a regulates target genes associated with various signaling pathways, including those of PI3K-Akt, MAPK, and GnRH. Thus, the present study findings indicate that miRNAs regulate the development of small intestines during the weaning process of piglets. 


\section{SUPPLEMENTARY MATERIALS}

Supplementary materials are only available online from: https://doi.org/10.5187/jast.2021.e69.

\section{REFERENCES}

1. Campbell JM, Crenshaw JD, Polo J. The biological stress of early weaned piglets. J Anim Sci Biotechnol. 2013;4:19. https://doi.org/10.1186/2049-1891-4-19

2. Le Dividich J, Sève B. Effects of underfeeding during the weaning period on growth, metabolism, and hormonal adjustments in the piglet. Domest Anim Endocrinol. 2000;19:63-74. https://doi.org/10.1016/S0739-7240(00)00067-9

3. Pluske JR, Hampson DJ, Williams IH. Factors influencing the structure and function of the small intestine in the weaned pig: a review. Livest Prod Sci. 1997;51:215-36. https://doi. org/10.1016/S0301-6226(97)00057-2

4. Drozdowski L, Thomson ABR. Intestinal mucosal adaptation. World J Gastroenterol. 2006;12:4614-27. https://doi.org/10.3748/wjg.v12.i29.4614

5. Groschwitz KR, Hogan SP. Intestinal barrier function: molecular regulation and disease pathogenesis.J Allergy Clin Immunol. 2009;124:3-20. https://doi.org/10.1016/j.jaci.2009.05.038

6. Wijtten PJA, van der Meulen J, Verstegen MWA. Intestinal barrier function and absorption in pigs after weaning: a review. Br J Nutr. 2011;105:967-81. https://doi.org/10.1017/ S0007114510005660

7. Meng Q, Sun S, Luo Z, Shi B, Shan A, Cheng B. Maternal dietary resveratrol alleviates weaning-associated diarrhea and intestinal inflammation in pig offspring by changing intestinal gene expression and microbiota. Food Funct. 2019;10:5626-43. https://doi.org/10.1039/ C9FO00637K

8. Kloosterman WP, Plasterk RHA. The diverse functions of microRNAs in animal development and disease. Dev Cell. 2006;11:441-50. https://doi.org/10.1016/j.devcel.2006.09.009

9. Osada H,Takahashi T. MicroRNAs in biological processes and carcinogenesis. Carcinogenesis. 2007;28:2-12. https://doi.org/10.1093/carcin/bgl185

10. Runtsch MC, Round JL, O'Connell RM. MicroRNAs and the regulation of intestinal homeostasis. Front Genet. 2014;5:347. https://doi.org/10.3389/fgene.2014.00347

11. Cui J, Zhou B, Ross SA, Zempleni J. Nutrition, microRNAs, and human health. Adv Nutr. 2017;8:105-12. https://doi.org/10.3945/an.116.013839

12. Pluske JR, Turpin DL, Kim JC. Gastrointestinal tract (gut) health in the young pig. Anim Nutr. 2018;4:187-96. https://doi.org/10.1016/j.aninu.2017.12.004

13. Lindemann MD, Cornelius SG, El Kandelgy SM, Moser RL, Pettigrew JE. Effect of age, weaning and diet on digestive enzyme levels in the piglet. J Anim Sci. 1986;62:1298-307. https://doi.org/10.2527/jas1986.6251298x

14. Cao ST, Wang CC, Wu H, Zhang QH, Jiao LF, Hu CH. Weaning disrupts intestinal antioxidant status, impairs intestinal barrier and mitochondrial function, and triggers mitophagy in piglets. J Anim Sci. 2018;96:1073-83. https://doi.org/10.1093/jas/skx062

15. Bailey M, Haverson K, Inman C, Harris C, Jones P, Corfield G, et al. The development of the mucosal immune system pre- and post-weaning: balancing regulatory and effector function. Proc Nutr Soc. 2005;64:451-7. https://doi.org/10.1079/PNS2005452

16. Inoue R, Tsukahara T, Nakatani M, Okutani M, Nishibayashi R, Ogawa S, et al. Weaning markedly affects transcriptome profiles and Peyer's patch development in piglet ileum. Front Immunol. 2015;6:630. https://doi.org/10.3389/fimmu.2015.00630 
17. Zhu LH, Xu JX, Zhu SW, Cai X, Yang SF, Chen XL, et al. Gene expression profiling analysis reveals weaning-induced cell cycle arrest and apoptosis in the small intestine of pigs. J Anim Sci. 2014;92:996-1006. https://doi.org/10.2527/jas.2013-7551

18. Bauer E, Metzler-Zebeli BU, Verstegen MWA, Mosenthin R. Intestinal gene expression in pigs: effects of reduced feed intake during weaning and potential impact of dietary components. Nutr Res Rev. 2011;24:155-75. https://doi.org/10.1017/S0954422411000047

19. Wang J, Chen L, Li P, Li X, Zhou H, Wang F, et al. Gene expression is altered in piglet small intestine by weaning and dietary glutamine supplementation. J Nutr. 2008;138:1025-32. https://doi.org/10.1093/jn/138.6.1025

20. Tao X, Xu Z. MicroRNA transcriptome in swine small intestine during weaning stress. PLOS ONE. 2013;8:e79343. https://doi.org/10.1371/journal.pone.0079343

21. Zou L, Xiong X, Wang K, Yin Y. MicroRNAs in the intestine: role in renewal, homeostasis, and inflammation. Curr Mol Med. 2018;18:190-8. https://doi.org/10.2174/156652401866618 0907163638

22. Ouyang M, Su W, Xiao L, Rao JN, Jiang L, Li Y, et al. Modulation by miR-29b of intestinal epithelium homoeostasis through the repression of menin translation. Biochem J. 2015;465:315-23. https://doi.org/10.1042/BJ20141028

23. Chiba M, Uehara H, Niiyama I, Kuwata H, Monzen S. Changes in miRNA expressions in the injured small intestine of mice following high-dose radiation exposure. Mol Med Rep. 2020;21:2452-8. https://doi.org/10.3892/mmr.2020.11054

24. Yu J, Liu F, Yin P, Zhu X, Cheng G, Wang N, et al. Integrating miRNA and mRNA expression profiles in response to heat stress-induced injury in rat small intestine. Funct Integr Genomics. 2011;11:203-13. https://doi.org/10.1007/s10142-010-0198-8

25. Cordes F, Brückner M, Lenz P, Veltman K, Glauben R, Siegmund B, et al. MicroRNA-320a strengthens intestinal barrier function and follows the course of experimental colitis. Inflamm Bowel Dis. 2016;22:2341-55. https://doi.org/10.1097/MIB.0000000000000917

26. Chen T, Xue H, Lin R, Huang Z. MiR-34c and PlncRNA1 mediated the function of intestinal epithelial barrier by regulating tight junction proteins in inflammatory bowel disease. Biochem Biophys Res Commun. 2017;486:6-13. https://doi.org/10.1016/j.bbrc.2017.01.115

27. Chen T, Xue H, Lin R, Huang Z. MiR-126 impairs the intestinal barrier function via inhibiting S1PR2 mediated activation of PI3K/AKT signaling pathway. Biochem Biophys Res Commun. 2017;494:427-32. https://doi.org/10.1016/j.bbrc.2017.03.043

28. Hampson DJ. Alterations in piglet small intestinal structure at weaning. Res Vet Sci. 1986;40:32-40. https://doi.org/10.1016/S0034-5288(18)30482-X

29. Montagne L, Boudry G, Favier C, Le Huërou-Luron I, Lallès JP, Sève B. Main intestinal markers associated with the changes in gut architecture and function in piglets after weaning. Br J Nutr. 2007;97:45-57. https://doi.org/10.1017/S000711450720580X

30. Shen Y, Gong JM, Zhou LL, Sheng JH. MiR-451 as a new tumor marker for gastric cancer. Oncotarget. 2017;8:56542-5. https://doi.org/10.18632/oncotarget.17239

31. Guo R, Gu J, Zhang Z, Wang Y, Gu C. MiR-451 promotes cell proliferation and metastasis in pancreatic cancer through targeting CAB39. BioMed Res Int. 2017;2017:2381482. https://doi. org/10.1155/2017/2381482

32. Zhong LN, Zhang YZ, Li H, Fu HL, Lv CX, Jia XJ. Overexpressed miR-196a accelerates osteogenic differentiation in osteoporotic mice via GNAS-dependent hedgehog signaling pathway.J Cell Biochem. 2019;120:19422-31. https://doi.org/10.1002/jcb.29166 